\title{
Propuesta de intervención sobre la violencia intrafamiliar: abordaje de acuerdo con la función y sentido del fenómeno violento presente en la dinámica familiar
}

\author{
Proposal of intervention on family violence: an approach \\ according to the function and sense of the violent \\ phenomenon in family dynamics
}

Germán Arley BAENA-VALLEJO'1 (iD) 0000-0003-1119-7630

Juan Gabriel CARMONA-OTÁLVARO'1 (ID) 0000-0002-5039-3514

Carlos Gustavo RENGIFO-ARIAS' ${ }^{1}$ (iD) 0000-0002-6645-2072

\begin{abstract}
Resumen
El artículo presenta una estrategia de intervención sobre la violencia intrafamiliar a partir de una resignificación conceptual de la noción de violencia y el planteamiento de su función y sentido. La violencia se entiende como una fuerza de carácter simbólico inherente al sistema familiar, que cumple la función de mantener, modificar o sustituir los vínculos, teniendo como efecto su reorganización. El sentido es abordado bajo las acepciones de significación, propósito y orientación que constituyen el carácter discursivo bajo el cual se manifiesta la violencia. La intervención tiene como base un modelo analítico que privilegia la palabra de los sujetos. Mediante el análisis, ellos comprenderán de qué manera su historia familiar, los lugares y funciones de sus integrantes, el modo de desempeñarlos y sus expectativas sobre aquellos, favorecen la expresión de la violencia como un modo de hacer frente a la falla propia de todo sistema familiar.
\end{abstract}

Palabras clave: Intervención; Sentido de coherencia; Violencia doméstica.

\footnotetext{
${ }^{1}$ Corporación Universitaria Minuto de Dios (UNIMINUTO), Facultad de Ciencias Humanas y Sociales, Programa de Psicología. Carrera 45, 22D-25, 051051, Bello, Antioquia, Colombia. Correspondence to: J.G. CARMONA-OTÁLVARO. E-mail: <jcarmon6@uniminuto.edu.co> Artículo elaborado como resultado de la investigación aprobada en la V Convocatoria para el Desarrollo y Fortalecimiento de la Investigación en UNIMINUTO, titulada "Intervención a la violencia Intrafamiliar: estrategia de abordaje sobre esta problemática social".

$\boldsymbol{\nabla} \boldsymbol{\nabla} \boldsymbol{\nabla}$

How to cite this article

Baena-Vallejo, G. A., Carmona-Otálvaro, J. G., \& Rengifo-Arias, C. G. (2020). Propuesta de intervención sobre la violencia intrafamiliar: abordaje de acuerdo con la función y sentido del fenómeno violento presente en la dinámica familiar. Estudos de Psicologia (Campinas), 37, e180104. http://dx.doi.org/10.1590/1982-0275202037e180104
} 


\begin{abstract}
The article presents a strategy for interventions on family violence, starting from a conceptual resignification of the notion of violence and a proposition regarding its function and meaning. Violence is conceived as a force of symbolic nature that is inherent to the family system and has the function of maintaining, modifying or substituting the bonds, thus resulting in their reorganization. Its sense is approached under the connotations of meaning, purpose, and orientation, that constitute the discursive nature which violence entails given its symbolic quality. The intervention is supported in an analytical model that privileges the subjects' words. Through the analysis, subjects will understand how their family history, the places and functions of its members, the way these are carried out, and their expectations about them, favor the expression of violence as a means of facing the failure of every familiar system.
\end{abstract}

Keywords: Intervention; Sense of coherence; Domestic violence.

La visibilización de la violencia intrafamiliar en las últimas décadas ha tenido como consecuencia la exacerbación del malestar social hacia este fenómeno. Las expresiones de maltrato que en épocas anteriores pasaban desapercibidas por la privacidad que se le asignaba a la realidad interior de la familia han adoptado un carácter execrable e inadmisible para la sociedad. Esta modificación en el modo como una sociedad asume la violencia y se comporta frente a ella obedece a que este fenómeno cuenta con una estructura simbólica que le permite ser maleable a la interpretación que una cultura particular le asigna; así, la violencia intrafamiliar constituye una construcción social que no es estática, por lo cual las conceptualizaciones y los modos de intervenirla varían a partir de los cambios culturales que experimenta la sociedad.

En el contexto colombiano, el fenómeno violento en la familia es reconocido como un problema social desde hace pocos años, pues aquello que sucedía bajo dicha connotación obedecía a la intimidad propia de la familia, y los actos que tenían el carácter de violentos eran validados o legitimados en virtud de la educación de los hijos, el control sobre el hogar, y como estrategia aceptada para solucionar los conflictos (Caicedo, 2005; Lugo \& Pablo, 2015). De esta época, caracterizada por una estructura patriarcal y jerarquizada, son propias varias representaciones y decires que configuraron el modo de asumir la violencia para la familia colombiana; frases como "la letra con sangre entra", soportadas en una tradición autoritaria que apelaba a la insensibilidad y la dureza en el carácter, dan cuenta de un modo de significar y asumir lo violento que le asignaba un carácter práctico y legítimo para la familia colombiana en épocas anteriores.

Así, es solo hasta la década de los noventa que la violencia intrafamiliar en Colombia deja de ser un asunto de interés privado para convertirse en un asunto de interés público (Caicedo, 2005), de modo que las políticas públicas referidas a este fenómeno solo aparecieron concretamente desde 1996 con la ley 294, que se encarga de desarrollar el artículo 42 de la Constitución Política y mediante el cual se dictan normas para prevenir, remediar y sancionar la violencia intrafamiliar.

Pero la visibilización de la violencia intrafamiliar como un asunto del cual el Estado debía encargarse solo surgió a partir de un proceso histórico que puede tener sus inicios en la Declaración de los Derechos de la Mujer y la Ciudadana en 1791, y que cobra mayor relevancia a partir de los movimientos feministas del siglo XIX y del siglo XX. Con estos movimientos, las mujeres inician un periodo histórico de reclamación frente a la violación de sus derechos (Caicedo, 2005; Toro, Rubio, \& Castro, 2017; Vázquez, 2012) y el acceso a la educación, al sufragio y a la remuneración igualitaria en el trabajo. Así, la mujer inicia un periodo de participación política en la transformación de las lógicas sociales, donde indudablemente prevalecían las estructuras patriarcales, jerarquizadas y donde el hombre ejercía una influencia notablemente mayor en las decisiones que correspondían a la organización de la sociedad (Nash, 2012).

El conjunto de sucesos que modificó el lugar y la influencia de las mujeres en el plano político y social halló legitimidad con eventos como la declaración del alto comisionado de las Naciones Unidas sobre la 
eliminación de la violencia contra la mujer en 1993, la elevación de la violencia contra la mujer como violación de los derechos humanos en la Conferencia de Viena en 1993 y la Convención de Belém do Pará en 1994. Dos años después, el Congreso de Colombia, como respuesta a estas declaraciones internacionales, inició el establecimiento de políticas públicas que estuvieran en función de prevenir, penalizar e intervenir el fenómeno violento en las familias. Se inició con la ley 294 ya mencionada, luego se estableció la ley 575 del año 2000 que otorgó funciones a las comisarías de familia para atender los casos de violencia intrafamiliar y se han hecho modificaciones en el código procedimental penal colombiano a partir de la ley 1542 de 2012 (Niño, 2014)

Es a partir de estas leyes que las instituciones públicas y privadas desarrollan programas que tienen el objetivo de intervenir la violencia intrafamiliar y de este modo disminuir el malestar social que dicho fenómeno produce. Sin embargo, a pesar de la visibilización social del fenómeno violento y la participación por parte del Estado en la tarea de intervenirlo, las cifras que anualmente publican las instituciones encargadas de su medición, como el Instituto Nacional de Medicina Legal y Ciencias Forenses (2018), siguen demostrando altos niveles de violencia intrafamiliar en el país, lo cual expresa no solo que el reconocimiento social de un fenómeno dispara las cifras de su manifestación, sino también que los modos que se utilizan actualmente para intervenir en él aún no cumplen con las expectativas que este fenómeno demanda (Téllez, Mancera, \& Vélez, 2016).

Este artículo de carácter reflexivo, pero que surge de un proceso de investigación de carácter interventivo, tiene el objetivo de presentar una propuesta de intervención sobre la violencia intrafamiliar a partir de un piso conceptual que se ha construido a través de tres fases de investigación. Es por ello que aquí pretendemos ofrecer una forma de concebir la violencia intrafamiliar diferente a la manera como habitualmente es definida; expondremos cuál es la función y el sentido de la violencia intrafamiliar, conceptos que consideramos imprescindibles para un abordaje riguroso de este fenómeno; y se presentará un modelo analítico de intervención que sustenta una estrategia concreta de intervención para la familia que se desarrollará al final de este artículo.

\section{Concepción de la violencia intrafamiliar, su función y sentido}

Un análisis que permita desentrañar la lógica interna de la violencia intrafamiliar exige una concepción previa sobre aquello que se entenderá por familia. En esta investigación, la familia se ha entendido a partir de una perspectiva estructural en su constitución, composición y funcionamiento. En su sentido más abstracto, la familia constituye un conjunto de elementos de carácter simbólico que se articulan entre sí a partir de determinados principios de estructura (Lévi-Strauss, Spiro, \& Gough, 1956; Benveniste, 1979). Estos principios de estructura son básicamente cuatro: incompletud del sistema, carencia de identidad propia de los elementos, covariancia de los elementos y dinamismo del sistema (Benveniste, 1979; Eidelsztein, 2008; Lévi-Strauss et al., 1956).

El sistema es incompleto ya que el registro simbólico que permite su articulación cuenta con esa característica; es decir, las posibilidades que tiene un sistema en su conformación están dadas por la naturaleza misma del lenguaje que, a pesar de contar con una multiplicidad de elementos utilizados para la significación, estos elementos no conforman una completud que permita decirlo todo (Eidelsztein, 2008). Por otra parte, los elementos de un sistema carecen de una identidad propia y definitiva ya que esta se encuentra supeditada a las relaciones y oposiciones que establece cada uno de los elementos con los demás miembros del sistema. Así, como ejemplo, un padre no adopta dicha función sino a partir de la relación y oposición que establece con quienes cumplen la función de madre y de hijo.

Esta característica de los elementos devela su modo de funcionamiento al interior del sistema que no es otro que la covariancia. Es decir, cada elemento está supeditado a su relación con los demás, de modo 
que el movimiento de uno afecta necesariamente a los demás elementos del sistema (Benveniste, 1979; Eidelsztein, 2008; Lévi-Strauss et al., 1956). Lo anterior le asigna al entramado simbólico un carácter dinámico permanente que es posibilitado por la identidad relativa de sus elementos y por la incompletud del sistema.

Esta perspectiva estructural de la familia permite comprender el funcionamiento de sus elementos y lo que sucede fenoménicamente entre los distintos integrantes físicos de la misma. Se habla de integrantes físicos pues, como se dijo, estos integrantes (padre, madre, hijo, hermano, etc.), representan los seres hablantes bajo los cuales se materializan, sin necesidad de correspondencia, las funciones simbólicas de un padre, una madre, un hijo, etc. Es por esta razón que el adjetivo "simbólico" pone en primer plano el orden lingüístico bajo el cual se articula la lógica de los vínculos familiares. Este grado de comprensión lleva a que se privilegie el análisis lógico del movimiento de los elementos para comprender su función, y posteriormente el sentido que, en su articulación con las representaciones sociales, se produce como interpretación singular de los actos violentos, bajo las acepciones de significación, propósito y orientación.

Es por lo anterior que, contrario al modo como habitualmente se conceptualiza la violencia intrafamiliar, como una fuerza aplicada sobre otro integrante de la familia que degrada o disminuye su valor con la intención de causarle daño (Jiménez-Rodrigo \& Guzmán-Ordaz, 2015; Rodríguez, 2018; Saliba, Wakayama, Moreira, Mendes de Paula, \& Isper, 2017) "y que tiene como efecto la ruptura de los vínculos que favorecen la adecuada convivencia con el otro o semejante" (Carmona \& Jaramillo, 2013, p.192), consideramos que un abordaje eficaz de este fenómeno requiere de una comprensión que se adentre en la lógica interna que posee la violencia en los vínculos familiares. Esta comprensión, que se diferencia de las concepciones descriptivas, estadísticas, y que sirven de base a sus explicaciones en las causas y los efectos (Costa et al., 2016; León, Grez, Prato, Torres, \& Ruiz, 2014), ha centrado su atención en desentrañar por qué una parte importante de las familias hacen uso de actos violentos en su cotidianidad; es decir, llama la atención que a pesar de ser un fenómeno que visiblemente es causante de malestar, sea una realidad cotidiana que experimentan muchos seres humanos al interior de sus familias.

Esta ruta de reflexión sobre el fenómeno violento, que se diferencia por lo tanto de las perspectivas predominantes, implica suspender la concepción convencional según la cual la violencia posee, como fin mismo, el propósito de generar daño, y seguir la sugerencia de Benjamin (1991), para quien el análisis de la violencia no debe apartarse del terreno de los medios. Así, al situar la violencia como un medio y no como una finalidad en sí misma, el análisis puede dirigirse al efecto lógico que se produce entre los elementos del sistema: ¿qué sucede con los vínculos cuando por lo menos uno de sus integrantes hace uso de la violencia? La pregunta así formulada ingresa al terreno matemático de la función, donde una operación que vincula a dos elementos de conjuntos distintos establece una relación particular entre ellos a partir de dicha función. Preguntarse así por la función de la violencia intrafamiliar corresponde a la necesidad de comprender qué sucede con los vínculos cuando estos experimentan la violencia (Martínez, Toro, \& Chavarria, 2016); de esta forma se suspende el análisis imaginario o representacional del cual es dotado el fenómeno violento, y se ingresa en el terreno estructural donde la familia, como se expresó anteriormente, se entiende como un sistema de carácter simbólico compuesto de elementos y funciones que interaccionan de forma covariante.

Desde esta perspectiva, concebimos la violencia como una fuerza de carácter simbólico, inherente a los vínculos que se establecen entre los elementos del sistema (en el caso de la violencia intrafamiliar el sistema será la familia) y que cumple la función de mantener, modificar o sustituir los vínculos, teniendo como efecto la reorganización de los mismos. Dicha reorganización es necesaria, pues dada la incompletud propia del sistema simbólico en el que se inscriben los miembros de la familia, esta misma corre el riesgo de desarticularse si las leyes y funciones que la comprenden no propician su cohesión, es decir, si dejan de cumplir el propósito de sostener el sentido que el conjunto de elementos constitutivos de la familia crearon para sostenerse como una familia. Cuando esto último sucede, se presentan las dificultades que ponen en 
peligro la subsistencia del sistema familiar, y es allí donde la violencia hace su aparición como una fuerza que, teñida de las características propias que ofrece un sistema simbólico (covariancia, no identidad propia, dinamismo, etc.), provoca que los elementos constitutivos del sistema se reorganicen con el propósito de no perder la cualidad de continuar siendo una estructura.

Pero la violencia, como un elemento dentro de la estructura y que posee una función, no siempre podrá cumplirla. Un sistema familiar se puede desarticular (si se entiende por ello, por ejemplo, una separación o ruptura), pues, aunque la violencia (diálogo, agresión, interdicción, silencios, etc.) opere para intentar reorganizar y mantener los vínculos, esta no siempre será suficiente. En tales casos, el vínculo no se mantiene, modifica o sustituye, sino que se rompe. Dicho de otra manera, lo que mantiene la cohesión de un sistema son sus leyes y las funciones que cumplen cada uno de los elementos. La violencia intenta subsanar la tentativa de desarticulación debido a la incompletud del sistema, pero si permanece un estado en el que los elementos no cumplen sus funciones o las leyes dejan de operar, entonces la violencia no bastará.

Dicho lo anterior, es posible plantear que la violencia es una fuerza de carácter simbólico que se cierne sobre una estructura, o los vínculos de los elementos de esta, con una función y sentido particular. Tratándose de la función, ella es doble: conserva la imagen o representación que los miembros del sistema poseen de sí, y a su vez, conserva el vínculo entre los mismos. El carácter particular que adopta el sistema es otorgado por tres operatorias que definen la doble función de la violencia: por medio del mantenimiento, modificación o sustitución de la imagen que posee de sí el hombre o mujer, padre, madre, hijo o hermano, se conserva ese tipo de representación (primera función) y, en consecuencia, a través de las mismas operatorias se conserva el vínculo entre los miembros del sistema (segunda función).

Por su parte, el sentido de la violencia intrafamiliar se inscribe en la experiencia singular bajo tres perspectivas: como significación, como propósito y como orientación. La primera acepción comprende aquello que los integrantes de una familia identifican como violencia; en muchos casos, un golpe no es asumido como violencia sino como muestra de reconocimiento para el otro (Carmona, Jaramillo, \& Garzón, 2015), lo cual da cuenta del modo como una familia representa el uso de esta fuerza. La segunda acepción consiste en el propósito de la violencia, es decir, el "para qué" del acto violento, que en la mayoría de los casos se encuentra vinculado al intento de recuperar o reestablecer la imagen que se percibe resentida a partir de la emergencia de las fallas del sistema familiar. La tercera acepción comprende la orientación, es decir, lo que sucederá con los vínculos familiares a partir del uso de la violencia y que pone de presente el modo como la violencia se inserta en la dinámica cotidiana de una familia. Estas tres acepciones sobre el sentido son necesarias en el estudio e intervención de la violencia, ya que se constituyen a partir del entramado de discursos que subyacen al actuar violento y que comprenden el primer material para poder acceder al contenido estructural; es decir, a la función de la violencia.

Para finalizar este apartado, de acuerdo con la perspectiva teórica que se expone, es posible hacer una crítica a las instituciones y programas de intervención que pretenden eliminar o erradicar la violencia, pues al ser una fuerza presente en la estructura lingüística creada por la cultura, es una realidad propiamente humana que no se puede eliminar (Sanmiguel, 1993). Tampoco debe confundirse con la agresividad que connota el ropaje imaginario del cual el ser hablante puede dotar a la fuerza violenta. Los actos agresivos en los seres humanos siempre tienen en su operación la respuesta a la vulneración de la imagen que psicológicamente constituye su yo, por lo cual es necesario aclarar que, si bien todo acto agresivo en la familia comprende un modo de expresión de la función reorganizadora de la violencia, no toda manifestación de la violencia se presenta por la vía de la agresividad. Un ejemplo de esta distinción teórica es que un golpe o insulto (acto agresivo) es un recurso que utiliza el ser humano cuando siente vulnerada la imagen de su yo o la que idealiza de otro; la violencia, como fuerza simbólica que posee una función en la estructura, procura reorganizar los vínculos mediante gestos, interdicciones, prohibiciones, diálogos y también actos agresivos. La agresividad, por lo tanto, es únicamente una forma de operación de la violencia. 


\section{Un modelo analítico para el abordaje de la violencia}

Para un abordaje de la violencia intrafamiliar que permita ingresar en el terreno de la lógica vincular entre los integrantes, es necesario que la base metodológica que guíe la intervención supere algunas debilidades presentes en las alternativas ofrecidas en la actualidad. En primer lugar, este abordaje debe superar la perspectiva de conciliación tal y como se ofrece en las Comisarías de Familia, ya que el privilegio del carácter legal solo permite abordar aspectos muy superficiales de los conflictos que no dan cuenta de la lógica vincular donde se cierne la violencia². En una vía similar a la anterior, el abordaje de la violencia no puede supeditarse a la penalización, pues esta alternativa, al igual que la anterior, impide que los familiares puedan comprender el entramado de sentidos que configuran su vinculación violenta y así poder realizar modificaciones; además de esto, y compartiendo una postura similar a la expuesta, el director del Instituto de Medicina Legal, Carlos Valdés, ha considerado que esta vía punitiva ha sido ineficaz en el abordaje de la violencia intrafamiliar (Valdés, 2017). En tercer lugar, es necesario que el abordaje sobre el fenómeno violento en las familias trascienda la perspectiva informativa que se encuentra en la base de muchos programas de promoción, prevención y sensibilización, pues en su base metodológica únicamente se halla el propósito de informar sobre contenidos preestablecidos y que generalmente no conciernen al malestar subjetivo ni a las experiencias que vivencian los participantes con sus propios familiares; en otras palabras, las familias participantes de estos programas pueden irse con la idea de que la violencia es algo lesivo para las relaciones humanas, que existen rutas específicas que se pueden seguir al vivenciar este fenómeno, que la comunicación es necesaria para disminuir o eliminar la violencia, y que las principales causas tienen que ver con los modos de crianza, el contexto social, el consumo de sustancias, el abuso de poder, entre otros aspectos; sin embargo, nada de ello puede explicarles lo que sucede en la lógica interna de sus vínculos, pues aquello que permite entender dicha lógica se halla en la narración misma de los individuos, de sus experiencias y del malestar inscrito en su relación con el otro, aspectos que no son privilegiados en ese modo de abordaje informativo presentado aquí como una debilidad de la intervención sobre la violencia intrafamiliar.

De acuerdo a lo anterior, es necesario que la base metodológica o modelo de intervención favorezca el uso de la palabra por parte de los sujetos que experimentan casos de violencia; con ello, es indispensable transmitir una actitud que ofrezca un viraje sobre el modo de asumir y afrontar la violencia, es decir, contrario a la actitud habitual de rechazo inmediato del fenómeno y de concebirlo como algo ajeno al ser humano, es menester propiciar en los sujetos una actitud de comprensión sobre su propia dinámica familiar, pues solo comprendiendo la lógica que en sus vínculos es favorecedora del uso de la violencia y los diversos sentidos con los cuales ella es utilizada, la familia podrá tener mayores posibilidades de introducir modificaciones en la base estructural de sus dificultades. Bajo estas características y con base en las concepciones teóricas antes expuestas, se implementó un modelo analítico de intervención que permitiera responder a la estructura simbólica que posee la violencia intrafamiliar y adquiriera la forma de una guía metodológica como estrategia concreta de intervención ${ }^{3}$.

\footnotetext{
V.

${ }^{2}$ En Colombia, las Comisarías de Familia son los organismos que a nivel municipal se encargan de recibir las denuncias por violencia intrafamiliar con el fin primordial de restablecer los derechos vulnerados hacia los integrantes. Desafortunadamente, la cantidad de casos supera por mucho el personal psicosocial y jurídico de estas instituciones; además, la perspectiva de conciliación como medida que procura no saturar los juzgados con procedimientos penales tiene como efecto un abordaje superficial que no favorece que los integrantes de una familia reconozcan las dinámicas que sustentan su modo de vinculación.

$\boldsymbol{\nabla} \boldsymbol{\nabla} \boldsymbol{\nabla}$

${ }^{3}$ El modelo analítico de intervención fue diseñado a partir de los planteamientos y conceptualizaciones desarrollados por el Grupo interinstitucional de investigación "El método analítico y sus aplicaciones en las ciencias sociales y humanas", el cual se encuentra adscrito a la Facultad de Ciencias Sociales y Humanas de la Universidad de Antioquia y a la Escuela de Humanidades de la Universidad EAFIT. El principal material se halla expuesto en los textos "El método analítico" (Lopera, Ramírez, Zuluaga, \& Ortiz, 2010) y "La vida como un juego existencial. Ensayitos” (Ramírez, 2012).
} 
La violencia intrafamiliar, al tener como base una estructura simbólica mediada por la lengua particular de una cultura, se encuentra inmersa en un entramado de decires que han configurado la historia de una familia, motivo por el cual, el modo más apropiado de afrontarla es por la vía de la palabra. En razón de lo anterior, este modelo analítico de intervención aplica como base metodológica el análisis del discurso, ya que mediante este proceso es posible que los integrantes de la familia resignifiquen los decires que sustentan la imagen que poseen de sí y de los otros, que configuran la lógica vincular, y que propician la emergencia de situaciones donde se hace uso de la violencia ante la expresión de las fallas propias de todo sistema familiar. Por esta razón, la intervención es concebida como un ejercicio de doble agente: por una parte, se encuentra lo que un profesional puede favorecer mediante preguntas, aclaraciones o indicaciones y, por otra parte, se encuentran los integrantes de la familia, quienes, en su función activa de comprensión, pueden intervenir en su propia estructura familiar.

Ahora bien, este modelo de intervención se apoya en una de las variantes del método científico que es el método analítico. Su carácter científico está dado por el propósito de cumplir con los criterios de consistencia y eficacia propios de la ciencia (Ramírez, 2012), los cuales son necesarios para establecer la validez de conjeturas o hipótesis. Para el caso de la intervención sobre la violencia intrafamiliar, su aplicabilidad se halla en la posibilidad de contrastar dialécticamente dos campos de la experiencia (Ramírez, 2012): la consistencia interna de los discursos que se basan en las tradiciones, creencias y concepciones particulares de los integrantes de la familia (teoría), y la eficacia de dichos discursos en la experiencia singular de la cotidianidad de los sujetos que se denominan "familia" (práctica). Para este propósito de carácter científico, el método analítico se presenta como "un camino para llegar a un resultado mediante la descomposición de un fenómeno en sus elementos constitutivos" (Lopera, Ramírez, Zuluaga, \& Ortiz, 2010, p.87). En el caso de la violencia intrafamiliar, se busca (resultado) que la familia pueda elaborar y contemplar otras maneras de resolver las manifestaciones de violencia que caracteriza sus relaciones.

En este modelo, el método analítico es aplicado sobre un discurso, es decir, sobre el conjunto de significados que configuran la realidad de un sujeto y de una familia, y que son el resultado del aprendizaje cultural (dimensión ideológica-moral), familiar (dimensión axiológica) y subjetivo (dimensión psíquica). Su aplicación se compone de cuatro procesos no necesariamente secuenciales: entender, criticar, contrastar e incorporar (Lopera et al., 2010; Ramírez, 2012). Entender consiste en "estudiar la coherencia lógica, las reglas de formación y los postulados explícitos e implícitos que fundamentan el discurso" (Ramírez, 2012, p.195); en el plano familiar, este proceso permite que los integrantes de la familia exploren los significados que subyacen a sus actos, y que en último término sustentan de manera consciente e inconsciente su posición subjetiva frente a la realidad familiar. Criticar obedece a una comparación entre los distintos discursos que un sujeto sostiene (crítica interna) y a una comparación entre el propio discurso con el sostenido por otros (crítica externa) (Ramírez, 2012). De acuerdo con este último autor, se trata de "estudiar la constelación discursiva y constatar si realmente se está de acuerdo con las teorías que se dice profesar" (Ramírez, 2012, p.196, cursivas del autor). De acuerdo con esto, los miembros de la familia, y ella misma en general, logran criticar sus discursos cuando permiten comparar entre sí las diversas posiciones que sus integrantes poseen respecto de una situación particular y favorecen la mutua transformación entre los diversos decires de la familia. Contrastar, por su parte, consiste en llevar al orden práctico lo que un discurso sostiene, con el fin de "verificar en la praxis la validez y las consecuencias del discurso: su eficacia y su fecundidad" (Ramírez, 2012, p.196, cursivas del autor); es decir, implica verificar si el discurso entendido y criticado por la familia funciona en la realidad de la experiencia a través de su puesta en práctica. Mediante este proceso, es posible determinar qué aspectos de carácter discursivo pueden modificarse a partir de los efectos que genera en los demás y en sí mismo. Por último, incorporar se refiere a un grado superior al proceso de entender, pues si este último alude al reconocimiento de la coherencia lógica de un discurso, la incorporación se refiere a la comprensión de ese discurso, es decir, "lograr que el texto sea asimilado, en primer lugar, por la teoría 
personal y, luego, por el propio cuerpo; convirtiéndose en parte de la actitud, del modo de ser: en un saber incorporado" (Ramírez, 2012, p.196, cursivas del autor). De este modo, la incorporación es un efecto de síntesis tras el proceso de análisis, pero que es vivido en el cuerpo en el sentido de que dicho discurso, de manera deliberada, se convierte en parte del modo de ser del sujeto. En el caso de la familia, un discurso es incorporado por ella cuando sus integrantes logran sentir con su cuerpo las implicaciones del discurso que los nombra como familia. Es importante aclarar que la incorporación es un punto de llegada en el análisis como también un punto de partida, puesto que son los discursos incorporados, pero no analizados, los que en muchas ocasiones constituyen la raíz moral que a la postre favorece el uso de la violencia.

Ahora bien, estos procesos que se acaban de describir se hallan favorecidos por tres componentes que el modelo de intervención busca transmitir mediante una actitud o proceso más general y que Ramírez denomina el quehacer del analítico (Lopera et al., 2010; Ramírez, 2012). Estos tres componentes son: escuchar, analizar e intervenir, los cuales complementan el ejercicio de los procesos denominados entender, criticar, contrastar e incorporar, puesto que son aplicables en cada uno de ellos. En otras palabras, para entender de una manera adecuada, es necesario escuchar, analizar e intervenir, y del mismo modo para criticar, contrastar, etc. Ahora bien, escuchar consiste en una "apertura al discurso del otro, una actitud de disposición que permita captar distintas manifestaciones discursivas, así como lo implícito y explícito que en ellas aparecen" (Lopera et al., 2010, p.159). Este componente es imprescindible para el modelo de intervención, en tanto la capacidad de escuchar y de disponerse a lo que el otro tiene para expresar es el requisito principal sin el cual no se podría llevar a cabo un auténtico análisis discursivo. En segundo lugar, analizar corresponde a los procesos expuestos anteriormente y que comprende la disposición para descomponer un discurso en sus elementos significantes. En tercer lugar, intervenir corresponde a "todas aquellas acciones que se realizan a partir de lo escuchado y que producen un efecto sobre el otro, sobre su discurso" (Lopera et al., 2010, p.164). Así, este proceso consiste, por lo expuesto, en un entrenamiento y un abordaje sobre sí mismo (la familia y cada uno de sus integrantes), que apoyado en la escucha y el análisis, permita que los actos o intervenciones sean cada vez más adecuadas para el sujeto, es decir, para cada integrante de la familia y para ella misma.

En resumen, la tarea metodológica que supone este modelo de intervención es transmitir una actitud o un modo de disposición de análisis sobre los actos violentos que vive la familia, una orientación que favorece la traducción en palabras a los afectos que en repetidas ocasiones operan sin ser verbalizados, manifestándose en actos que fracturan la dinámica familiar y, que al ofrecer la oportunidad de nombrar adecuadamente el malestar subjetivo que le subyace, pueda ser un movilizador de la potencia creativa que permita la exploración de nuevas formas de afrontar las fallas inherentes al sistema familiar.

\section{Estrategia de intervención sobre la violencia intrafamiliar}

Una resignificación sobre la manera como se comprende y se explica el fenómeno violento en la familia tiene una incidencia directa en el modo como esta se interviene; por esta razón, con base en los planteamientos conceptuales antes expuestos, esta estrategia de intervención dista teóricamente de la mayoría de propuestas ofrecidas en la actualidad en tanto asume, en primer lugar, que no se debe apuntar a la eliminación de la violencia sino al favorecimiento de su comprensión (por qué y para qué se presenta) $y$, en segundo lugar, que la familia al poseer un entramado de funciones y sentidos que le han permitido configurarse como tal, cuenta con recursos básicos que le pueden permitir explorar vías diferentes donde no se privilegie la manifestación agresiva en la función reorganizadora de la violencia.

Dicho de otro modo, dado que la violencia es concebida como una fuerza que al interior de una estructura realiza una operación lógica de reorganización con el propósito de conservar los vínculos, será la 
manifestación agresiva (insultos, golpes, silencios, etc.) la que deberá menguarse, y este propósito, consideramos, puede conseguirlo una familia mediante la comprensión de su lógica vincular y mediante el resarcimiento y reelaboración de las funciones y sentidos que han operado a favor de la articulación del sistema familiar. La apuesta, por lo tanto, no consiste en el aislamiento o punición de los integrantes por la expresión de la violencia. La propuesta apunta a la exhortación para que la familia haga frente responsablemente a las dificultades que no le son extrañas a un sistema incompleto como este.

En razón de lo anterior, esta estrategia de intervención consiste en un dispositivo basado en el modelo analítico antes expuesto, "donde se privilegia la palabra de los sujetos implicados en el fenómeno violento, con el propósito de que estos, mediante la descomposición de sus decires, comprendan de qué manera su historia familiar, los lugares y funciones de sus integrantes, el modo de desempeñarlos y sus expectativas sobre aquellos, favorece la expresión de la violencia como un modo de hacer frente a la falla propia del sistema que constituye la familia. Tras este proceso comprensivo, la estrategia busca que los integrantes de la familia tejan en conjunto nuevas formas de resolver y asumir responsablemente las dificultades propias de sus vínculos familiares".

La estrategia se puede desarrollar bajo dos modalidades de intervención: una en la cual un profesional cumple la función de facilitador en la intervención que realiza la familia y otra donde los integrantes de la familia intervienen sobre ella sin el acompañamiento de un profesional. Para el primer caso, que puede ser desarrollado por instituciones estatales o privadas a través de psicólogos o terapeutas familiares, la estrategia se sirve de una "Guía de Intervención" (Carmona, Baena, \& Rengifo, 2018b) dirigida principalmente a los profesionales, donde se presenta la base teórica y metodológica necesaria para llevar a cabo la función que, desde nuestra perspectiva, debería llevar a cabo el profesional que pretenda favorecer los propósitos de intervención descritos. Esta es fundamental, puesto que orienta al profesional sobre las mismas cualidades que deberá desarrollar para que la intervención sea lo más eficaz posible y le muestra las limitaciones mismas que puede poseer la intervención.

Para el segundo caso, la estrategia se materializa en una Cartilla de intervención sobre la violencia intrafamiliar (Carmona, Baena, \& Rengifo, 2018a), en la cual se desarrolla todo el dispositivo de intervención que se describirá a continuación y que constituye nuestra apuesta por una intervención de la familia sobre sí misma. Sobre esta modalidad, se hace fundamental la voluntad estatal o privada, por ejemplo, en instituciones educativas, para facilitar el acceso de las familias a este material.

La cartilla se compone de cinco momentos que se apoyan metodológicamente en el modelo analítico de intervención. Cada uno de ellos cuenta con actividades individuales y grupales basadas en los cuatro procesos del método analítico (entender, criticar, contrastar e incorporar), y en las tres actitudes generales de dicho método (escuchar, analizar e intervenir) aplicadas a cada uno de los procesos. El dispositivo ofrece inicialmente un proceso de reconocimiento de la historia familiar y de la lógica vincular de sus integrantes, luego ingresa al terreno de las dificultades familiares y finaliza con el diseño de nuevas estrategias de afrontamiento de las dificultades por parte de la familia y un seguimiento o evaluación de todo el proceso.

Todo este proceso está orientado en la cartilla mediante textos breves que introducen los momentos y las actividades, pero que implícitamente transmiten a la familia aspectos elementales de la lógica de la violencia que se convierten en un estímulo para favorecer el análisis. En las actividades grupales, la cartilla se sirve de mensajes cortos que tienen la función de favorecer la transmisión de las tres actitudes analíticas, para que, con ello, se dé prioridad a la verbalización del material implícito al discurso familiar. Además de lo anterior, cada momento finaliza con un breve escrito y con una serie de preguntas que tienen el propósito particular de indagar por el sentido de la violencia en las tres acepciones antes descritas: significación, propósito y orientación. 
Primer momento: "Nuestra familia. ¿Cómo nos hicimos familia?" Este eslabón tiene el propósito de que los integrantes puedan reconocer la historia que subyace a su construcción como familia pues, como se ha dicho, la expresión de la violencia mediante actos agresivos es únicamente la capa más superficial de un entramado que halla sus raíces en los discursos, experiencias y decisiones que configuran la historia familiar; en otras palabras, el acontecer presente es un resultado de todo un proceso histórico, que al ser verbalizado favorece el reconocimiento y la comprensión de la lógica vincular actual de la familia.

Segundo momento: "Nuestro lugar en la familia. ¿Quién soy en y para mi familia?" En este momento se pretende que los integrantes, apoyados en la historia rememorada de su conformación, logren reconocer el lugar que desempeñan al interior de su familia, el modo cómo lo desempeñan y las exigencias que subyacen de cada integrante hacia esos lugares. Aunque no de manera explícita, el abordaje de la violencia intrafamiliar ingresa aquí por la vía de la imagen o representación de sí, y es este reconocimiento el que propiciará el carácter subjetivo de la responsabilidad que se posee en el favorecimiento de la lógica conflictiva en los vínculos. En otras palabras, ya que la agresividad constituye uno de los velos más utilizados como expresión de la violencia en las familias, es la imagen de cada integrante la que se privilegia cuando al sentirla vulnerada, se reacciona agresivamente en un intento de resarcirla, validarla o refrendarla. Así, lo que se exige idealmente de sí mismo y de los demás en sus diversas funciones (padre, madre, hija, abuela, tío, etc.), constituye la cuota imaginaria que fácilmente se desliza hacia un favorecimiento propio, haciendo uso de la agresividad para conservar una imagen y un modo de vínculo ideal para sí.

Tercer momento: "Las dificultades en nuestra familia. ¿Por qué y para qué se presentan?" En este momento ingresa la violencia intrafamiliar de manera explícita ya que se indaga expresamente por cómo la imagen que se posee de sí y de los demás es generadora de dificultades y cuál es el propósito que poseen los actos que se llevan a cabo para responder a esas dificultades. El propósito que posee este momento de la intervención es que los integrantes consigan verbalizar los sentidos (significación, propósito y orientación) inscritos en el actuar violento, de modo tal que el reconocimiento del contenido imaginario que se pretende conservar por la vía de la agresión les ofrezca una mayor comprensión sobre la función que posee la violencia en la lógica vincular que han construido y les permita adquirir otro tipo de herramientas para intervenir sobre dicha forma de relacionarse.

Cuarto momento: "Las respuestas ante las dificultades de nuestra familia. ¿Cómo resolvemos los problemas familiares?" Este momento constituye el ejercicio explícito de pensar modos diferentes a los habituales de solucionar las dificultades de la familia. Tanto en la actividad individual como en la grupal, se pretende que los integrantes de la familia hagan uso del saber incorporado acerca de su historia, sus lugares y su modo cotidiano de resolver las dificultades, en el propósito de construir alternativas diferentes a las que comprenden el uso de actos agresivos. En este momento, se hace un especial énfasis en privilegiar el bienestar de la familia por sobre el interés individual, ya que históricamente la conformación y mantenimiento de la familia ha requerido de diversas renuncias por parte de quienes la integran (Lévi-Strauss et al., 1956). Para el caso de la violencia intrafamiliar, dichas renuncias responderían en la mayoría de las ocasiones a la imagen que se exige de sí y del otro. Pero privilegiar el bienestar de la familia no es apropiado sin el uso de la deliberación y del análisis; por ello, el momento también invita a que los integrantes incorporen, en su modo de resolver las dificultades, la capacidad inherente al ser humano que consiste en el uso de la razón.

Quinto momento: "Nuestros logros y retos. ¿Qué hemos construido y qué podemos seguir modificando?" Este momento constituye el cierre de la intervención y, a diferencia de los anteriores momentos, no cuenta con dos actividades sino únicamente con una de carácter grupal. El propósito principal de este momento es que la familia realice un recorrido por todo el proceso de intervención, rememorando las modificaciones que ha conseguido y los aspectos que aún se pueden modificar; además de ello, se busca que los integrantes comprendan que las dificultades al afectar la dinámica familiar, no son responsabilidad únicamente de quienes 
las propician o las favorecen, sino de todo el conjunto de miembros de la familia, razón por la cual todos tendrán la posibilidad de asumir una posición que la favorezca.

Debido a lo anterior, no se trata de transmitir la idea de una familia ideal que se autoregula constantemente favoreciendo la emergencia de un bienestar individual y colectivo, sino de comprender que, ante las dificultades, cada uno tiene la capacidad de elegir cómo incidirá y que, para esto, abrirse al otro por medio de la escucha e intervenir mediante el uso de la palabra razonada, puede incrementar las posibilidades de tomar decisiones que favorezcan al sistema familiar.

\section{Conclusión}

Con este artículo hemos pretendido ofrecer y poner en discusión estos planteamientos y esta forma de intervenir la violencia intrafamiliar, especialmente, porque consideramos que es función de la academia, y particularmente en nuestro país, trazar nuevas alternativas de concebir el fenómeno violento que apunten a una mayor comprensión de su funcionamiento. Su abordaje no puede estar alejado del estudio y del diseño de propuestas interdisciplinares, pues la violencia intrafamiliar no constituye un fenómeno que se deje adscribir a una sola disciplina y, los estudios que así lo han hecho, generalmente aportan únicamente un aspecto de su manifestación; así, la violencia posee un carácter múltiple y complejo, por lo cual su abordaje debe responder a las mismas características de dicho fenómeno. El reto consistirá en que los entes gubernamentales, los centros de investigación y las instituciones correspondientes, puedan centrar su atención y sus recursos en abordajes e intervenciones novedosas que puedan incidir de manera eficaz en la lógica del fenómeno violento presente en las dinámicas familiares.

Por otra parte, reconocemos que una limitación importante de nuestra investigación es que todavía no se ha llevado a cabo un proceso de pilotaje y una fase de intervención propiamente dicha que ponga en ejecución el modelo y la propuesta aquí expuesta. Luego de la finalización de esta tercera fase de investigación, estamos a la expectativa de una cuarta fase interventiva que permita evaluar los efectos de esta propuesta en las dos modalidades de intervención. También esperamos que con la publicación de la Guía y la Cartilla de Intervención, otros profesionales también interesados en la violencia intrafamiliar puedan ponerla en uso y contribuir al mejoramiento de las formas de comprensión y de abordaje de esta realidad humana.

Para finalizar, es importante resaltar que nuestra propuesta no pretende una naturalización de la violencia (término utilizado por algunos para explicar la desensibilización de los colombianos ante los actos que han trazado nuestra historia como Nación) ni mucho menos justificarla; nuestro propósito consiste en mostrar que la violencia es una realidad exclusivamente humana y que su abordaje eficaz no puede consistir en pasarla por alto, responder con una cuota de agresividad o censurarla indefectiblemente. Es menester explorar su modo de funcionamiento, la lógica estructural que se esconde tras las palabras y actos que la representan y optar por el favorecimiento de la serena deliberación que propenda por la articulación más que por la disolución.

\section{Colaboradores}

Todos los autores participaron en el diseño, ejecución y análisis de la información de cada uno de los productos de investigación, incluido el presente material. 


\section{Referencias}

Benjamin, W. (1991). Para una crítica de la violencia y otros ensayos. Madrid: Taurus.

Benveniste, E. (1979). Problemas de lingüística general I. Ciudad de México: Siglo Veintiuno.

Caicedo, C. (2005). Lucha contra la violencia intrafamiliar: Perspectivas desde la experiencia colombiana. In C. C. Caicedo, P. Haeberli, \& B. Defrance (Orgs.), Les droits de l'homme, l'interdit de la violence scolaire et familiale (pp.71-97). París: Asociación mundial Escuela Instrumento de Paz. Recuperado de http://www.cifedhop.org/Fr/Publications/Thematique/ thematique13/Caicedo.pdf

Carmona J., \& Jaramillo J. (2013). Análisis de las perspectivas teóricas sobre la noción de violencia, y su relación con la violencia intrafamiliar. Katharsis, 16, 181-196.

Carmona, J., Jaramillo, J., \& Garzón, D. (2015). Función de la violencia intrafamiliar entre cónyuges (Padres de Familia). Katharsis, 20, 101-121.

Carmona, J., Baena, G., \& Rengifo, C. (2018a). Cartilla de intervención sobre la violencia intrafamiliar. Bogotá: Corporación Universitaria Minuto de Dios.

Carmona, J., Baena, G., \& Rengifo, C. (2018b). Guía de intervención sobre la violencia intrafamiliar. Bogotá: Corporación Universitaria Minuto de Dios.

Costa, N., Gomes, H., Almeida, T., Pinheiro, R., Almeida, C., Gondim, L., ... Lima, V. (2016). Violence against women: can "jealousy" mitigate the significance of violence? Estudos de Psicologia (Campinas), 33(3), 525-533. http://dx.doi. org/10.1590/1982-02752016000300015

Eidelsztein, A. (2008). Las estructuras clínicas a partir de Lacan I: intervalo, holofrase, locura, psicosis, psicosomática y debilidad mental. Buenos Aires: Letra Viva.

Instituto Nacional de Medicina Legal y Ciencias Forenses. (2018). Forensis 2017: datos para la vida. Colombia: Autor.

Jiménez-Rodrigo, M. L., \& Guzmán-Ordaz, R. (2015). The kaleidoscope of violence against women in union based on gender inequality: a review of analytical approaches. Revista de Estudios Sociales, 2015(54), 93-106. http://dx.doi. org/10.7440/res54.2015.07

León, T., Grez, M., Prato, J. A., Torres R., \& Ruiz, S. (2014). Violencia intrafamiliar en Chile y su impacto en la salud: Una revisión sistemática. Revista Médica de Chile, 142(8), 1014-1022. http://dx.doi.org/10.4067/\$0034-98872014000800009

Lévi-Strauss, C., Spiro, M., \& Gough, K. (1956). Polémica sobre el origen y la universalidad de la familia. Barcelona: Anagrama.

Lopera, J., Ramírez, C., Zuluaga, M., \& Ortiz, J. (2010). El método analítico. Medellín: Universidad de Antioquia.

Lugo, V., \& Pablo, J. (2015). Antropología y "estudios de la violencia" en Colombia: en busca de una perspectiva crítica. Revista Colombiana de Antropología, 51(1), 245-269. Recuperado de http://www.scielo.org.co/scielo.php?script=sci_ arttext\&pid=S0486-65252015000100010 \&lng=en\&tIng=es

Martínez, N. Y., Toro, M. I. O., \& Chavarria, E. F. V. (2016). Aspectos subjetivos relacionados con la violencia intrafamiliar. Caso municipio de Sabaneta Antioquia. Estudios Sociales, 24-25(47), 348-376. Recuperado de http://www.scielo. org.mx/scielo.php?script=sci_arttext\&pid=S0188-45572016000100348\&lng=es\&tlng=es.

Nash, M. (2012). Mujeres en el mundo: historia, retos y movimientos. Madrid: Alianza.

Niño, M. (2014). Estado del arte de la violencia intrafamiliar en Colombia: estudios socio-jurídicos. Revista de Derecho Público, 33, 4-29. Recuperado de https://derechopublico.uniandes.edu.co/components/com_revista/archivos/derechopub/ pub471.pdf

Ramírez, C. (2012). La vida como un juego existencial. Ensayitos. Medellín: Fondo Editorial Universidad EAFIT.

Rodríguez, J. M. V. (2018). Violence, peace and environmental conflicts in Colombia: an overview from political ecology and the sociology of violence. Revista Luna Azul, 46, 409-421. http://dx.doi.org/10.17151/luaz.2018.46.21

Saliba, C., Wakayama, B., Moreira, R., Mendes de Paula, A., \& Isper, A. (2017). La violencia intrafamiliar y los procesos notificatorios bajo la óptica del profesional de salud pública. Revista Cubana de Salud Pública, 43(2), 204-213. Recuperado de http://scielo.sld.cu/scielo.php?script=sci_arttext\&pid=S0864-34662017000200007\&lng=es\&tlng=es

Sanmiguel, P. (1993). Consideraciones previas al estudio de la violencia. Revista Colombiana de Psicología, 2, 81-87. Recuperado de http://www.revistas.unal.edu.co/ index.php/psicologia/article/view/15786/16601

Téllez, M., Mancera, N., \& Vélez, P. (2016). Aplicación de la enseñanza clínica del derecho en casos de violencia intrafamiliar. Revista Indisciplinas, 2(3). Recuperado de http://www.unaula.edu.co/sites/default/files/la\%20ense\%c3\%b1anza\%20 cl\%c3\%adnica\%20del\%20derecho\%20a\%20partirv2n4.pdf 
Toro, B. L., Rubio, L. O., \& Castro, J. F. (2017). Gender violence has no frontiers. comparative study about colombian and spain violence social standards of genders (2004-2014). Revista Derecho del Estado, 38, 127-154. http://dx.doi. org/10.18601/01229893.n38.05

Valdés, C. (julio 1, 2017). Entrevista por Semana [online]. En Colombia la violencia no desaparece, se transforma. Semana, Colombia. Recuperado de http://www.semana.com/nacion/articulo/carlos-valdes-director-del-instituto-de-medicinalegal-violencia-en-colombia/511538

Vázquez, M. (2012). La violencia de género, el papel de los movimientos feministas y los posibles abordajes jurídicos. Revista de la Facultad de Derecho, 33. 95-111. Recuperado de http://revista.fder.edu.uy/index.php/rfd/article/view/6/6

Recibido: Septiembre 18, 2018

Versión final: Octubre 9, 2019

Aprobado: Octubre 23, 2019 